\title{
Temporal Shifts in Antibiotic Resistance Elements Govern Virus-Pathogen Conflicts
}

\author{
Kristen N. LeGault ${ }^{1}$, Stephanie G. Hays ${ }^{1}$, Angus Angermeyer ${ }^{1}$, Amelia C. McKitterick ${ }^{1}$, Fatema-tuz Johura ${ }^{2}$, \\ Marzia Sultana ${ }^{2}$, Munirul Alam² and Kimberley D. Seed ${ }^{1,3 *}$
}

\author{
Affiliations: \\ ${ }^{1}$ Department of Plant and Microbial Biology, University of California, Berkeley, Berkeley, CA 94720, USA \\ ${ }^{2}$ icddr,b, formerly International Centre for Diarrhoeal Disease Research, Dhaka, Bangladesh \\ ${ }^{3}$ Chan Zuckerberg Biohub, San Francisco, CA 94158, USA \\ * Correspondence to: kseed@berkeley.edu
}

\begin{abstract}
:
Bacteriophage predation selects for diverse anti-phage systems that frequently cluster on mobilizable defense islands in bacterial genomes. However, there remains a lack of molecular insight into the reciprocal dynamics of phage-bacterial adaptations in nature, particularly in clinical contexts where there is need to inform phage therapy efforts and understand how phages drive pathogen evolution. Here, using time-shift experiments we show that fluctuations in SXT integrative and conjugative elements (ICEs), which notoriously confer antibiotic resistance, govern Vibrio cholerae's susceptibility to phages in clinical samples. We find that SXT ICEs, which are widespread in Gammaproteobacteria, invariably encode phage defense and function to protect other genera from phage attack following conjugation. We discover phage counter-adaptation to SXT-mediated restriction in clinical samples, and show that heterogeneity in SXT ICEs allows for re-emergence of phage resistance. Further, phage infection stimulates high frequency SXT ICE conjugation, leading to the concurrent dissemination of phage and antibiotic resistance.
\end{abstract}




\section{Main Text:}

Bacteriophage (phage) predation has been estimated to account for upwards of $40 \%$ of all bacterial mortality (1), shaping microbial community composition and selecting for bacteria that evade predation $(2,3)$. Coevolution between phages and their hosts contributes to global biodiversity $(2,4-6)$. Indeed, genetic differences between closely related strains are largely phage resistance genes (7), with anti-phage defense systems comprising upwards of $10 \%$ of a bacterial genome (8), and mutations that decrease host susceptibility accounting for further inter-strain variability $(9,10)$. Phylogenetic analyses show that defense systems associate with mobilome genes, indicating that phage predation can drive bacterial evolution through mobile genetic element (MGE) flux $(8,11)$. A recent explosion in the identification of novel defense systems $(11,12)$ has allowed for a re-assessment of bacterial genomes, identifying resistance determinants in genetic contexts not previously associated with phage defense.

Despite the central role of phages in microbial evolution and ecology, there remains a dearth of biologically relevant systems to study ongoing phage-host interactions in natural environments. Approaches to study phage resistance systems often rely on ectopic expression of candidate systems in heterologous bacterial hosts and assessing restriction of phages that are not known to have encountered the system in nature. Although this approach has proven lucrative for the discovery of novel defense systems (11-14) and is especially valuable for assessing genes from unculturable organisms or those without known phages, this approach precludes our understanding of fluctuating interactions and competition between defense systems, as well as the consequences and trade-offs of phage resistance. Furthermore, the extent to which a given defense system is expressed in its native context as well as the potential for dissemination of resistance to naïve hosts through horizontal transfer cannot be assessed. These approaches also do not support the discovery of phage-encoded counter defenses, which lag behind our knowledge of anti-phage defenses but are likely key drivers of defense diversification.

A promising model system to address unanswered questions in phage-host ecology and evolution lies in the long-term sampling of Vibrio cholerae and its lytic phages (vibriophages). Pathogenic V. cholerae strains of the O1 serogroup possessing the key virulence factor cholera toxin are responsible for the infectious diarrheal disease cholera, which continues to pose a significant global public health threat (15). In Bangladesh, where cholera is endemic, lytic phage predation is implicated in modifying the duration and severity of cholera outbreaks $(16,17)$. Interestingly, cholera patients can shed both vibriophages and viable $V$. cholerae, indicating that complex interactions between phages and their hosts take place within the human intestine, as neither entity is driven to extinction. Long-term sampling of cholera-infected patients in Bangladesh has revealed the persistence of three dominant lytic phages ICP1, ICP2 and ICP3 (39), making this a powerful system to understand biologically relevant outcomes of phage predation and to discover reciprocal adaptations in phages infecting pathogenic bacteria within the context of human disease.

\section{SXT ICEs determine clinical Vibrio cholerae phage susceptibility}

To gain insight into the ongoing co-evolutionary dynamics between $V$. cholerae and its phages, we evaluated whether clinical $V$. cholerae isolates were susceptible to infection by ICP1 from past, future or contemporaneous patient samples (Fig. 1A). A pattern emerged in which $V$. cholerae isolates were uniformly susceptible to contemporaneous phage, but restricted ICP1 from temporally mismatched patients (Fig. 1B). $V$. cholerae isolates from the past were susceptible to phages from this time period, but restricted infection of phage from future patient samples, while isolates from 2018 onward restricted phages from earlier sampling dates but were susceptible to contemporaneous phages (Fig. 1B). Importantly, $V$. cholerae DCP66 from December 2017 was uniquely susceptible to all ICP1 isolates, suggesting it lacks a phage resistance determinant, and phages from this sample were uniquely restricted by all other isolates of $V$. cholerae.

Differences in $V$. cholerae's susceptibility to ICP1 could not be explained by known anti-phage systems. However, comparative genomics of clinical $V$. cholerae isolates from this time period revealed the variable presence and identity of integrative and conjugative element (ICEs) of the SXT/R391 family (18). These 
$\sim 100 \mathrm{~kb}$ islands are of clinical importance for pathogens such as Proteus mirabilis and $V$. cholerae, conferring antibiotic resistance to sulfamethoxazole, trimethoprim (SXT) and, variably, streptomycin, tetracycline, kanamycin and chloramphenicol $(19,20)$. SXT ICEs are composed of core and accessory genes, with the core genes encoding functions related to SXT regulation and transmission through conjugative transfer (Fig. 1D). Accessory genes, such as those conferring resistance to heavy metals or antibiotics, determine the identity of the SXT ICE and are clustered in five "hotspot" regions (19) (Fig. 1D). The temporal pattern of phage resistance observed during this sampling period correlated with the transition of ICE $V$ chInd6(+) to SXT(-) to ICEVchInd5(+) $V$. cholerae, suggesting that SXT ICEs may confer protection against clinically relevant vibriophages. To test whether SXT ICEs can confer phage resistance, we conjugated ICE $V c h$ Ind6 and ICE $V$ chInd5 into the phage sensitive $V$. cholerae SXT(-) strain E7946. Each SXT ICE was sufficient to inhibit plaque formation by ICP1 recovered during the sampling period (Fig. 1C). These results demonstrate that SXT ICEs found in $V$. cholerae natively express a resistance determinant sufficient to restrict vibriophage cocirculating with SXT ICEs in cholera patients.

The genes predicted to be responsible for phage resistance in ICE $V c h$ Ind5 and ICE $V c h$ Ind6 were readily identified, and in both ICEs the genes localized to hotspot 5. ICE VchInd5 hotspot 5 contains a type 1 bacteriophage exclusion (BREX) system, an abundant phage resistance system that restricts phages through an unknown mechanism known to rely on epigenetic modification (14, 21). Additionally, ICE $V c h$ Ind5 hotspot 5 encodes a putative abortive infection gene (Abi), and a gene of unknown function (Fig. 1D). ICEVchInd6 contains several restriction-modification (RM) systems as well as hypothetical genes in hotspot 5 (Fig. 1D). Deletion of hotspot 5 from ICE $V c h$ Ind5 and ICE $V c h$ Ind6 rendered $V$. cholerae susceptible to ICP1 infection, demonstrating that the anti-phage systems in hotspot 5 are necessary for phage resistance (Fig. 1C). On the basis of these results, we examined hotspot 5 regions in all published SXT ICEs by supplementing data from ICEBerg 2.0 (22) with additional published SXT ICEs since the database was last updated. Strikingly, we observed that all 76 de-duplicated SXT ICE genomes published to date harbor predicted anti-phage systems in the same hotspot 5 location, with the exception of three elements that had no discernible hotspot 5, suggesting it was lost through recombination. All hotspot 5 regions share a putative transcriptional regulator possessing a WYL-domain previously shown to associate with defense systems (23) (Fig. 1D). A phylogenetic tree based off of the nucleotide sequence of the WYL-domain gene clustered SXT ICEs based on the type of anti-phage system encoded in hotspot 5, building a tree that does not agree with the relationship inferred from the conservation of the gene downstream of hotspot $5 \mathrm{traI}$, suggesting that hotspot 5 cargo genes move "en bloc" (Fig. S1). This demonstrates that gene cargo dedicated to phage defense is a ubiquitous yet unrecognized facet of the biology of SXT ICEs (Fig, 1D). Hence, although SXT ICEs are infamous for their role in the spread of antibiotic resistance, these elements also encode for and thus disseminate phage resistance throughout Gammaproteobacteria, including in human pathogens like Proteus mirabilis and $V$. cholerae for which phage therapy/prophylaxis has been proposed (24-26).

To ascertain the global relevance of SXT ICE-mediated phage resistance in $V$. cholerae, we determined the identity and prevalence of SXT ICEs in published toxigenic O1 $V$. cholerae genomes, and in a collection of 247 isolates sequenced as a part of this study. Genomes of 2,000 isolates were analyzed with isolation dates going back to 1987 when SXT was first identified in toxigenic V. cholerae (27), to late 2019 (Fig. 1E). Previous analysis of 96 SXT ICEs from seventh pandemic $V$. cholerae isolates identified a total of five different SXT ICEs in toxigenic $V$. cholerae, and found that ICEVchInd5 dominated globally (28). Our expanded analysis demonstrates that ICE $V c h$ Ind5 remains globally dominant, followed by ICEVchInd6 and ICEVchBan9 (also known as ICEVchMoz10) (Fig. 1E). Additional SXT ICEs are found as singletons in toxigenic V. cholerae: MO10 (the first discovered and best characterized SXT ICE) and ICEVchInd4 (also known as ICE $V c h$ Ban11) (Fig. 1E). Of note, we found an additional SXT ICE, ICEPmiUSA1, not previously described in $V$. cholerae which contains genes in hotspot 5 with PFAM domains characteristic of the DISARM system (13) (Fig 1D and 1E). Despite the obvious selective advantage afforded by SXT ICEs in conferring antibiotic resistance in $V$. cholerae, we also find that SXT(-) toxigenic O1 strains persist through this sampling period.

\section{Genetic dissection of SXT ICE hotspot 5-mediated restriction of phages and MGEs}


To gain further insight into how SXT ICEs influence $V$. cholerae ecology and gene flux, we tested the ability of SXT ICEs to restrict foreign genetic elements that are clinically important to $V$. cholerae. We focused on the three most prevalent SXT ICEs in toxigenic $V$. cholerae for further study, ICEVchInd5, ICE $V c h$ Ind6 and ICE $V c h$ Ban9, which contain either a BREX system, or several restriction-modification systems in hotspot 5 (Fig. 2A). V. cholerae exconjugants possessing each SXT ICE were challenged with the three most prevalent lytic vibriophages found in cholera patient stool samples: ICP2, ICP3 (both Podoviridae) and two temporally distinct isolates of ICP1 (Myoviridae). Each SXT ICE tested conferred resistance to some, but not all, of the vibriophages tested (Fig. 2B, Fig. S2). Although ICEVchInd5 and ICEVchBan9 both encode type 1 BREX systems, they showed different restriction profiles with regards to ICP1, likely because they possess highly divergent genes encoding for $\operatorname{BrxX}$ (26\% amino acid identity), the essential methyltransferase and restriction determinant for BREX (14).

We next wanted to determine which system is responsible for phage inhibition in $V$. cholerae, and whether different restriction enzymes restrict different phages. From ICE $V c h$ Ind6, each restriction enzyme was deleted, while the methyltransferase $b r x X$ was deleted from ICE $V c h$ Ind5 and ICE $V c h B a n 9$. ICE $V c h$ Ind5 $\Delta b r x X$ and ICE $V c h$ Ban9 $\Delta b r x X$ no longer inhibited ICP1 and ICP3, showing that the BREX system is responsible for inhibition of lytic vibriophages (Fig. 2B). Deletion of each putative restriction enzyme in ICEVchInd6 showed that the protein with homology to BstXI (protein ID AIM16524.1), a type II restriction enzyme, is largely responsible for ICEVchInd6's anti-phage activity, however, there were differences between ICP1 isolates (Fig. 2B). Specifically, ICE $V c h$ Ind6 $\Delta b s t X I$ was susceptible to ICP $1^{2006}$, yet ICP $1^{2017}$ was still partially inhibited. Deletion of the type I restriction enzyme $h s d R$ also had differential effects on the two isolates of ICP1 tested. ICE $V c h$ Ind6 $\Delta h s d R$ still completely inhibited ICP $1^{2006}$, but ICP $1^{2017}$ was able to spontaneously overcome ICE $V c h$ Ind6 $\Delta h s d R$ with plaquing that did not scale with the dilution series (Fig. S3), suggesting that HsdR has some activity against ICP1 $1^{2017}$, which notably co-circulated with ICEVchInd6(+) V. cholerae.

Next, we asked whether SXT ICEs restrict other MGEs, including temperate phage that can provide beneficial traits to their hosts upon integration into the bacterial chromosome. Specifically, we assessed whether SXT ICEs restrict acquisition of two clinically important and beneficial MGEs in epidemic $V$. cholerae: the temperate phage CTX $\phi$ and the phage-inducible chromosomal island like elements (PLEs). The filamentous phage CTX $\phi$ encodes cholera toxin, and is essential for $V$. cholerae's pathogenicity in the human host (29). PLEs are $\sim 20 \mathrm{~kb}$ phage satellites that confer resistance to ICP1 and have been a persistent part of the mobilome of epidemic $V$. cholerae dating back to at least 1949, before SXT was common in clinical $V$. cholerae isolates (30). The acquisition of antibiotic marked CTX $\phi$ and each PLE was assessed in otherwise isogenic $V$. cholerae hosts either lacking an SXT ICE or harboring each of the most prevalent SXT ICEs (ICEVchInd6, ICE $V c h$ Ind5, ICE $V$ chBan9), or derivatives lacking hotspot 5 (Fig. 2C and 2E). SXT ICEs restricted acquisition of both PLEs and $\mathrm{CTX} \phi$, but to varying extents, and in all cases, transduction was restored in mutants lacking hotspot 5 (Fig. $2 \mathrm{D}$ and $2 \mathrm{~F}$ ). PLE 1 was uniquely restricted by ICEVchInd6 compared to PLEs 2, 3, 4 and 5 (Fig $2 \mathrm{~F}$ and Fig. S4B-E). The difference in inhibition of different PLEs can be explained by the predicted recognition motif for BstXI from Bacillus subtilis, which shares 50\% amino acid identity with ICEVchInd6's BstXI homolog: CCANNNNN^NTGG ((31)), which is found once in PLE 1 but not in other PLEs. Knocking out this site restored PLE 1 transduction to the level observed for the SXT(-) host (Fig. S4A), again demonstrating the relative contribution of BstXI to ICE $V c h$ Ind6's restriction of foreign elements. Collectively these data demonstrate that like other anti-phage defense systems, SXT-mediated restriction of MGEs comes at a cost. While all SXT ICEs restricted infection by clinically relevant lytic vibriophages, they also limited acquisition of CTX $\phi$ and PLEs, which provide beneficial traits to epidemic $V$. cholerae, observations that help to explain the maintenance of SXT(-) strains in nature (Fig. 1E).

\section{SXT ICEs disseminate phage resistance to other Gammaproteobacteria}

SXT ICEs are recombinogenic, self-transmissible mobile elements hypothesized to evolve by genetic exchange of variable and hotspot regions $(28,32)$ suggesting that gene cargo in hotspot 5 could be mobilized 
between taxa through both SXT ICE conjugation and through recombination of hotspot 5 with other SXT ICEs or homologous DNA. To determine the extent of lateral dissemination, genes in hotspot 5 from ICEVchInd6, ICE $V c h$ Ind5 and ICE $V c h$ Ban9 were queried using BLASTn to probe whether the genes could be found in other taxa, either within SXT ICEs or in a different genetic context. The genes in ICE $V$ chInd6 hotspot 5 were always localized to an SXT ICE, and ICEVchInd6 in its entirety is found in other Gammaproteobacteria with over 99\% nucleotide identity (Fig. 3A, Table S3). Intriguingly, a variant of ICEVchInd6 was also identified in Pseudoalteromonas sp. referred to as ICEPspSpa1(33). ICEPspSpa1 is 96.45\% identical to ICEVchInd6 except in place of bstXI is a SIR2 domain protein that is implicated in the Thoeris defense system (11) (Fig. 3A).

Despite being globally dominant in toxigenic $V$. cholerae O1 strains, the ICE $V$ chInd5 BREX system was rarely found outside of $V$. cholerae, occurring in hotspot 5 from an otherwise divergent SXT ICE in P. mirabilis and outside of an MGE in Shewanella (Fig. 3A and Table S4). The BREX system from ICEVchBan9 was the most widely distributed, both taxonomically and in terms of genetic context, being found within SXT ICEs (for example, in Escherichia coli and P. mirabilis), as well as outside of SXT ICEs. These varied locations include the chromosome, on plasmids, as well as in non-SXT ICEs (Fig. 3A and Table S5). The distribution of hotspot 5 encoded anti-phage systems in other Gammaproteobacteria suggests that these systems confer resistance to a broad range of phages infecting different hosts well beyond $V$. cholerae, and that the self-transmissible nature of SXT ICEs disseminates these anti-phage systems to new taxa.

The test whether SXT ICEs can confer phage resistance upon entry into a new host, ICEVchInd6, ICE $V c h$ Ind5 and ICE $V c h$ Ban9 and the cognate $\Delta$ hotspot 5 derivatives, were conjugated into E. coli (Fig. 3B) and exconjugants were challenged with the lytic coliphages T2, T4, T5, T6 and T7. Strikingly, all SXT ICEs tested offered protection from at least one coliphage, with the broadest protection afforded by ICEVchInd6 (restricting all phage except for T5) and the narrowest restriction afforded by ICE $V$ chInd5 (which only partially restricted T2) (Fig. 3C, Fig. S5). ICEVchBan9, which is natively found in E. coli (Fig 3A), restricted T2, T5 and T6, while T4 and T7 were not inhibited. The T7-encoded DNA mimic protein, Ocr, was recently shown to inhibit type 1 BREX (34) and thus may explain T7's resistance to ICEVchBan9 and ICEVchInd5. E.coli exconjugants harboring the $\triangle$ hotspot 5 SXT ICE derivatives were susceptible to all phages tested, demonstrating that it is the same anti-phage systems in hotspot 5 that confers resistance in E.coli and $V$. cholerae. This demonstrates that upon acquisition of SXT ICEs through conjugation, hotspot 5 is natively expressed in the recipient taxa and confers resistance to their cognate phage.

\section{Co-Evolution of Vibriophage ICP1 with VchInd5}

In light of the discovery that $\mathrm{SXT}(+) V$. cholerae restricts phages through anti-phage systems known to rely on differential epigenetic modification and subsequent restriction, we postulate that phages shed from patients infected with SXT ICE $(+) V$. cholerae have escaped restriction through stochastic acquisition of DNA modification. Epigenetically modified phage can then productively infect co-circulating strains possessing the same SXT ICE, but are unable to infect $V$. cholerae with a different SXT ICE (as shown in our time-shift assay, Fig. 1B). In further support of this, phage in the patient stool in which we recovered SXT ICE(-) V. cholerae DCP66 were restricted by all SXT ICE(+) hosts, and the SXT ICE(-) $V$. cholerae isolate from this patient was uniquely susceptible to infection by phages from all patient stool samples (Fig. 1B). Other genetic means of overcoming modification-based restriction are common among phages and include anti-restriction proteins, modification of DNA bases and loss of recognition motifs (reviewed in (35). To distinguish between potential mechanisms of epigenetic or genetic escape, ICP1 isolates from patient stool samples were first passaged on SXT ICE(-) V. cholerae, and then evaluated for the ability to productively infect the clinical $V$. cholerae strains from our original time-shift experiment (Fig. 4A). ICP1 isolates from stool samples in which we co-recovered ICE $V$ chInd6(+) $V$. cholerae lost the ability to productively infect isolates with ICE $V c h$ Ind6, suggesting that ICP1 shed in patient stool samples had escaped through acquiring modification during the course of infection (Fig. 4A). In contrast, the pattern of susceptibility seen with ICEVchInd5 and cognate phages indicates a genetic route of escape. Specifically, ICP1 shed from patient stool samples with $V$. cholerae harboring ICE $V$ chInd5 retained the ability to infect co-circulating ICEVchInd5(+) isolates after passage through an SXT(-) host, yet 

available under aCC-BY-ND 4.0 International license.

ICP1 isolates shed in patient samples in which we recovered ICE $V$ chInd6 $(+) V$. cholerae were still restricted by ICEVchInd5 (Fig. 4A). This was reminiscent of the phenotype for ICP1 ${ }^{2006}$, which was not restricted by ICEVchInd5 (Fig. 2B). To identify the genetic mechanism responsible for the host range differences between ICP1 isolates, we compared the genomes of ICP1 isolates from across a temporal gradient that can and cannot infect $V$. cholerae harboring ICEVchInd5 (Fig. 4B). This analysis revealed differences in the operon encoding gene products (gp) 21-25 that co-varied with plaque formation on a ICE $V$ chInd5(+) host (Fig. 4B and 4C). Unlike ICP1 isolates that can productively infect ICEVchInd5(+) V. cholerae, the restricted ICP1 ${ }^{2017}$ lacks both gp21 and the promoter region for $g p 25$, which we identified by analyzing the ICP1 transcriptome (61) (Fig. 4C), suggesting one of these early genes may allow ICP1 to overcome restriction by ICE $V c h$ Ind5. Having narrowed in on these two gene products as candidate anti-ICE $V$ chInd5 proteins, we evaluated if in trans expression of either $g p 21$ or $g p 25$ was sufficient to rescue plaque formation for the restricted ICP ${ }^{2017}$ phage. Ectopic expression of $g p 25$, but not $g p 21$, restored ICP $1^{2017}$ 's ability to form plaques on $V$. cholerae harboring ICEVchInd5 (Fig. 4D), supporting our designation of gp25 as orbA for "overcome restriction by BREX". To decipher if OrbA was the sole factor permitting ICP1 plaque formation on the ICE $V$ chInd5 $(+)$ host, we expressed $o r b A$ during ICP3 infection, since ICP3 is an unrelated phage that is also restricted by the BREX system in ICE $V c h$ Ind5 (Fig. 2B). Here too we observed that $\operatorname{orb} A$ expression restored productive infection for ICP3 on the ICEVchInd5(+) host (Fig. 4D). These results demonstrate that OrbA (YP_004250966) functions as an anti-BREX protein with activity against ICE $V c h$ Ind5. Notably, ICE $V c h$ Ind5 does not restrict ICP2, CTX $\phi$ nor the majority of $E$. coli phages tested, even though these genomes possess the ICEVchInd5 methylation site previously identified by PacBio sequencing as RTAAAYG (36) (Table S6). Although the mechanism of BREX restriction is not known, restriction relies on detection of unmethylated motifs (21). This suggests the other unrestricted phages and MGEs tested here have mechanisms to overcome ICEVchInd5, though they do not have homologs of $\operatorname{orb} A$. OrbA encodes a small 44 amino acid protein with no homology to any annotated proteins, as well as no PFAM/DUF domains. Furthermore, PSI-BLAST did not return any significant homologs outside of ICP1. The T7-encoded anti-restriction protein Ocr has been shown to overcome BREX in E. coli by functioning as DNA mimic (34). Although the crystal structure for OrbA has not been solved, a predicted model does not show the electrostatics characteristic of a DNA mimic like Ocr (Fig. S6), suggesting a different mechanism of action for OrbA's anti-BREX activity. Our findings also indicate that OrbA is not a broadly active anti-BREX protein since the BREX system encoded by ICE $V$ chBan9 equally restricts ICP $1^{2006}$ and $\operatorname{ICP} 1^{2017}$ (Fig. 2B).

We next set out to document the reciprocal dynamics between the repertoire of SXT ICEs in $V$. cholerae with anti-BREX activity afforded by OrbA in ICP1 in greater detail. Towards this end, we assessed the presence and identity of SXT ICEs in 467 isolates of toxigenic O1 V. cholerae from Bangladesh spanning 2001-2019. During this time period we observed fluctuating dominance of ICE $V c h$ Ind5 and ICEVchInd6 (Fig, S7). To probe the relationship between phage predation and transitions in SXT ICE prevalence, we analyzed a subset of $151 \mathrm{~V}$. cholerae and 44 ICP1 isolates from November 2016 to September 2019 collected and sequenced from cholera patient samples as a part of this study (Fig. 4E). As was evident in the subset of samples in which ICP1 and $V$. cholerae were co-isolated (Fig. 1B), we observed a transition between circulating ICE $V c h$ Ind6 $(+)$ strains to ICE $V c h I n d 5(+)$ strains in 2018, with intermittent recovery of SXT ICE(-) $V$. cholerae from patients between June 2017 and May 2018. Interestingly, ICP1 isolates lacking the $\operatorname{orb} A$ promoter region (as in ICP1 ${ }^{2017}$ (Fig. 4C)) were only isolated during a time period when ICE $V c h$ Ind5 was absent in cholera patients (Fig. 4E); however, concurrent with the emergence of $V$. cholerae ICE $V c h I n d 5(+)$ in patient stool samples in the spring of 2018, all contemporary ICP1 isolates possess the promoter for $o r b A$. This locus provides an example of selection for a novel anti-BREX gene found in phage, which can counter restriction by an SXT ICE, and shows how temporal dynamics in SXT ICEs can exert purifying selection on phages. In times when ICP1 is not encountering ICE $V c h$ Ind5 in nature, the locus that confers resistance to ICE $V c h$ Ind5 is not under positive selection. Thus, variants with promoter mutations persist, or are perhaps selected for, suggesting this locus provides no fitness benefit to ICP1 when it is not co-circulating with ICEVchInd5(+) V. cholerae.

\section{Phage infection stimulates SXT ICE conjugation}


SXT ICEs are SOS responsive, meaning that DNA damage leading to the accumulation of single stranded DNA triggers de-repression of SXT, culminating in SXT excision and expression of operons related to conjugative transfer (37). DNA damaging agents such as mitomycin C (MMC), ultraviolet radiation, and certain classes of antibiotics that interfere with DNA replication (for example, ciprofloxacin), have been shown to increase SXT conjugation frequency (38). DNA damage may serve as a proxy for impaired cell viability, thus SXT ICEs that can mobilize and spread horizontally to escape a compromised host would have a selective advantage. We postulated that cell damage from infection by lytic phages could serve as an input into SXT ICE de-repression and transfer. To test this hypothesis, we evaluated the frequency of conjugative transfer of ICE $V c h$ Ind5 into recipient $V$. cholerae following infection by either ICP $1^{2006}$ or ICP $1^{2017}$, representing phages that are not restricted or are fully restricted by ICEVchInd5, respectively (Fig 2B). ICEVchInd5 $(+) V$. cholerae was infected with ICP1 at a multiplicity of infection of 0.5 , and frequency of transfer into a differentially marked $V$. cholerae recipient was determined. Importantly, the added differentially marked $V$. cholerae recipient lacks the $\mathrm{O} 1$ antigen, which is the receptor for ICP1 (39), making the recipient insensitive to ICP1 infection, yet proficient for conjugation (Fig. S8). As expected, conjugation frequency was elevated above background following treatment with only MMC (Fig. 5A). Interestingly, infection by ICP $1^{2006}$ (but not the restricted phage ICP $1^{2017}$ ) resulted in high frequency SXT ICE transfer compared to an uninfected control, comparable to conjugation frequency observed from a MMC treated donor (Fig. 5A). This increase in conjugation frequency per donor cell was driven by a consistent frequency of transfer despite productive phage infection among the donor strains resulting in a 100-fold decrease in donor cell viability, yet remarkably, constant transfer frequency (Fig. S9). As expected, no antibiotic resistant exconjugants were detected with a $\triangle \operatorname{traD}$ donor, demonstrating that phage-mediated transduction is not a contributing factor in these assays (Fig. $5 \mathrm{~A})$. It is unclear whether it is the infected cells that are conjugating at a greater frequency, or if the uninfected cells in the population are sensing phage lysis through an unknown mechanism and becoming high frequency donors. We propose alternative models, the first where cellular damage wrought by phage infection triggers increased transfer of SXT ICEs as the SXT ICE races to transfer out of a dying host (Fig. 5B), or, alternatively, productive phage infection in neighboring cells leads to increased conjugative transfer from uninfected cells, through a yet undescribed mechanism.

\section{Discussion}

One of the prominent genomic features of current seventh pandemic $V$. cholerae are ICEs of the SXT/R391 family, infamous for conferring multidrug resistance $(18,19,28)$. SXT ICEs appeared in sequenced epidemic $V$. cholerae in 1987 and currently dominate in clinical isolates in regions where cholera is endemic $(27,40,41)$. Here we show that SXT ICEs invariably encode phage-resistance genes in hotspot 5 , linking together antibiotic and phage resistances on a single mobile genetic element widespread in

Gammaproteobacteria. This builds on a previous study which showed the SXT ICEVspPor3 reduced plaquing by the phage T1 when conjugated into E. coli (42), however, it was not demonstrated that this was due to hotspot 5, or whether ICEVsp Por3 offered protection against cognate phages in its native host context.

Co-evolution between $V$. cholerae and lytic vibriophages is driven, at least in part, by presence and identity of SXT ICEs. Using a time-shift experiment (43), we find that lytic phage can infect contemporary $V$. cholerae hosts but cannot infect hosts from the past or the future. This pattern is consistent with negative frequency-dependent dynamics, where the faster evolving partner (here, lytic phage) has higher fitness in the presence of the contemporary antagonist than those from the past or the future (44-46). However, over a short time scale, co-evolution of ICP1 with $V$. cholerae appears to be driven by selective sweeps. Adaptations that allow for phage infection of contemporary $\mathrm{SXT} \operatorname{ICE}(+) V$. cholerae sweep to fixation over short times scales, rendering contemporary ICP1 more fit than ICP1 from the near past, which is characteristic of arms race dynamics (45-47). ICP1's adaptations to overcome contemporary SXT ICEs is driven both by changes in its capacity to express the anti-ICE $V c h$ Ind5 BREX gene product $\operatorname{orb} A$ and acquisition of epigenetic modification allowing for infection of ICE $V c h$ Ind6 hosts. The heterogeneity in ICE SXT identity and presence in circulating toxigenic $V$. cholerae suggests the potential for population bottlenecks and subsequent sweeps. Phages that have 

available under aCC-BY-ND 4.0 International license.

acquired anti-SXT ICE mechanisms will sweep through the population, providing a selective advantage to hosts with SXT ICEs that confer resistance to the dominant phages. The subsequent sweep of the resistant SXT ICE through the $V$. cholerae population generates a further bottleneck on the ICP1 population. These dynamics of fluctuating genomic dominance in a population of bacteria and phages allows for apparent long-term coexistence between phages and their bacterial hosts, where neither entity drives the other to extinction. The heterogeneity in SXT ICE-mediated phage defense can be considered a "pan-immune system" (2), where the collective pool of SXT ICEs circulating in $V$. cholerae serve to restrict all lytic phages: ICP1, ICP2 and ICP3. Additionally, these temporal dynamics provide insight into the ongoing maintenance of SXT(-) V. cholerae, which should have a selective disadvantage within the clinical context since it lacks the antibiotic resistances conferred by SXT ICEs. However, SXT(-) V. cholerae provide a population-wide benefit, as phage that have propagated on an SXT(-) host would lack appropriate epigenetic modifications for subsequent infection of SXT $\mathrm{ICE}(+)$ hosts, allowing for persistence of $V$. cholerae. The prevalence of diverse anti-phage systems carried by other SXT ICEs suggests that these dynamics play out in other Gammaproteobacteria.

The ability SXT ICEs to restrict beneficial mobile genetic elements such as CTX $\phi$ and PLEs has implications on bacterial evolvability. Each SXT ICE tested restricted transduction of some, but not all, elements tested, linking the relative abundance of a given MGE or prophage to the prevalence of a restrictive SXT ICE in the population, and further demonstrating the extent to which phage predation can alter the pool of genes in a population. The ability for SXT ICEs to restrict MGEs and phages shows a tradeoff that may favor SXT(-) V. cholerae in certain conditions, such as in changing environments (48). SXT ICEs create a barrier to genetic exchange from cells possessing a different SXT ICE (or no SXT ICE), as only DNA modified due to activity of the same hotspot 5-encoded modification-based restriction system will be permitted as it would be indistinguishable from self, which could result in genetic divergence between closely related strains. Indeed, comparative genomics has shown that genetic exchange between genomes is higher if they encode cognate RM systems (49). Coupled with earlier findings that ICEVchInd5 can limit natural transformation in $V$. cholerae through a hotspot 4 encoded DNase (50), the role of SXT ICEs in controlling bacterial gene flux is likely multifaceted.

The human gut may represent an environment where SXT ICE conjugation can occur between gut commensals or invading pathogens, especially when DNA damaging antibiotics such as ciprofloxacin have been administered, as ciprofloxacin has been both shown to increase SXT conjugation through activation of the SOS response (38) and is frequently secreted from cholera patients (51). Here we have shown that phage infection can also increase the frequency of SXT conjugation in $V$. cholerae. Interestingly, infection by a phage that is not restricted by ICE $V c h$ Ind5 stimulated higher frequency conjugation, while infection by a restricted phage did not. There is an expected energetic cost and accompanying growth delay associated with the formation of the conjugative machinery, as has been shown for other ICEs (52). When infected by a restricted phage, there may be little benefit for SXT ICEs to trigger conjugation, as their host cell may suffer a selective disadvantage limiting the SXT ICE's vertical transmission. However, during productive phage infection, an SXT ICE that can escape a dying cell would have be able to move laterally. Together, we can now appreciate that both antibiotics and phages can simultaneously spread the genes for antibiotic resistance and phage resistance by stimulating the transfer of SXT ICEs. As we continue to grapple with increasing multidrug resistant bacterial infections, which have garnered a renewed interest in phage therapy, it is of critical importance that we work to understand how phages and antibiotics interact in driving the evolution of dangerous pathogens like $V$. cholerae. 


\section{References}

1. Wilhelm,S.W. and Suttle,C. a (1999) Viruses and Nutrient Cycles in the Sea aquatic food webs. Bioscience, 49, 781-788.

2. Bernheim,A. and Sorek,R. The pan-immune system of bacteria: antiviral defence as a community resource. https://doi.org/10.1038/s41579-019-0278-2

3. Koskella,B. (2019) New approaches to characterizing bacteria-phage interactions in microbial communities and microbiomes. Environ. Microbiol. Rep., 11, 15-16.

https://doi.org/10.1111/1758-2229.12706

http://www.ncbi.nlm.nih.gov/pubmed/30431224

4. Brockhurst,M.A., Chapman,T., King,K.C., Mank,J.E., Paterson,S. and Hurst,G.D.D. Darwin review Running with the Red Queen: the role of biotic conflicts in evolution. 10.1098/rspb.2014.1382.

https://doi.org/10.1098/rspb.2014.1382

5. Koskella,B. and Brockhurst,M.A. (2014) Bacteria-phage coevolution as a driver of ecological and evolutionary processes in microbial communities. FEMS Microbiol. Rev., 38, 916-931.

https://doi.org/10.1111/1574-6976.12072

http://www.ncbi.nlm.nih.gov/pubmed/24617569

6. Paterson,S., Vogwill,T., Buckling,A., Benmayor,R., Spiers,A.J., Thomson,N.R., Quail,M., Smith,F., Walker,D., Libberton,B., et al. (2010) Antagonistic coevolution accelerates molecular evolution. Nature, 464, 275-278.

https://doi.org/10.1038/nature08798

http://www.ncbi.nlm.nih.gov/pubmed/20182425

7. Rodriguez-Valera,F., Martin-Cuadrado,A.B., Rodriguez-Brito,B., Pašić,L., Thingstad,T.F., Rohwer,F. and Mira,A. (2009) Explaining microbial population genomics through phage predation. Nat. Rev. Microbiol., 7 , 828-836.

https://doi.org/10.1038/nrmicro2235

http://www.ncbi.nlm.nih.gov/pubmed/19834481

8. Koonin,E. V, Makarova,K.S. and Wolf,Y.I. (2017) Evolutionary Genomics of Defense Systems in Archaea and Bacteria *. Annu. Rev. Microbiol, 71, 233-61.

https://doi.org/10.1146/annurev-micro-090816

9. Seed,K.D., Yen,M., Jesse Shapiro,B., Hilaire,I.J., Charles,R.C., Teng,J.E., Ivers,L.C., Boncy,J., Harris,J.B. and Camilli,A. (2014) Evolutionary consequences of intra-patient phage predation on microbial populations.

Elife, 3, 1-10.

https://doi.org/10.7554/eLife.03497

10. Zheng,Y., Roberts,R.J. and Kasif,S. (2004) Identification of genes with fast-evolving regions in microbial genomes. Nucleic Acids Res., 32, 6347-6357.

https://doi.org/10.1093/nar/gkh935

http://www.ncbi.nlm.nih.gov/pubmed/15576679

11. Doron,S., Melamed,S., Ofir,G., Leavitt,A., Lopatina,A., Keren,M., Amitai,G. and Sorek,R. (2018)

Systematic discovery of antiphage defense systems in the microbial pangenome. Science (80-. )., 359.

https://doi.org/10.1126/science.aar4120

12. Gao,L., Altae-Tran,H., Böhning,F., Makarova,K.S., Segel,M., Schmid-Burgk,J.L., Koob,J., Wolf,Y.I.,

Koonin,E. V. and Zhang,F. (2020) Diverse enzymatic activities mediate antiviral immunity in prokaryotes.

Science (80-. )., 369, 1077-1084.

https://doi.org/10.1126/science.aba0372

http://www.ncbi.nlm.nih.gov/pubmed/32855333

13. Ofir,G., Melamed,S., Sberro,H., Mukamel,Z., Silverman,S., Yaakov,G., Doron,S. and Sorek,R. (2017) 
DISARM is a widespread bacterial defence system with broad anti-phage activities. Nat. Microbiol.,

10.1038/s41564-017-0051-0.

https://doi.org/10.1038/s41564-017-0051-0

http://www.ncbi.nlm.nih.gov/pubmed/29085076

14. Goldfarb,T., Sberro,H., Weinstock,E., Cohen,O., Doron,S., Charpak-Amikam,Y., Afik,S., Ofir,G. and Sorek,R. (2015) BREX is a novel phage resistance system widespread in microbial genomes. EMBO J., 34, $169-183$.

https://doi.org/10.15252/embj

15. Ali,M., Nelson,A.R., Lena Lopez,A. and Sack,D.A. (2015) Updated Global Burden of Cholera in Endemic Countries. 10.1371/journal.pntd.0003832.

https://doi.org/10.1371/journal.pntd.0003832

16. Faruque,S.M., Islam,M.J., Ahmad,Q.S., Faruque,A.S.G., Sack,D.A., Nair,G.B. and Mekalanos,J.J. (2005) Self-limiting nature of seasonal cholera epidemics: Role of host-mediated amplification of phage. Proc. Natl. Acad. Sci., 102, 6119-6124.

https://doi.org/10.1073/pnas.0502069102

http://www.ncbi.nlm.nih.gov/pubmed/15829587

17. Jensen,M.A., Faruque,S.M., Mekalanos,J.J. and Levin,B.R. (2006) Modeling the role of bacteriophage in the control of cholera outbreaks. Proc. Natl. Acad. Sci. U. S. A., 103, 4652-4657.

https://doi.org/10.1073/pnas.0600166103

http://www.ncbi.nlm.nih.gov/pubmed/16537404

18. Waldor,M.K., Tschäpe,H. and Mekalanos,J.J. (1996) A new type of conjugative transposon encodes

resistance to sulfamethoxazole, trimethoprim, and streptomycin in Vibrio cholerae O139. J. Bacteriol., 178,

4157-4165.

https://doi.org/10.1128/jb.178.14.4157-4165.1996

http://www.ncbi.nlm.nih.gov/pubmed/8763944

19. Wozniak,R.A.F., Fouts,D.E., Spagnoletti,M., Colombo,M.M. and Ceccarelli,D. (2009) Comparative ICE Genomics: Insights into the Evolution of the SXT/R391 Family of ICEs. PLoS Genet, 5, 1000786.

https://doi.org/10.1371/journal.pgen.1000786

20. Li,X., Du,Y., Du,P., Dai,H., Fang,Y., Li,Z., Lv,N., Zhu,B., Kan,B. and Wang,D. (2016) SXT/R391

integrative and conjugative elements in Proteus species reveal abundant genetic diversity and multidrug resistance OPEN. Nat. Publ. Gr., 10.1038/srep37372.

https://doi.org/10.1038/srep37372

21. Gordeeva,J., Morozova,N., Sierro,N., Isaev,A., Sinkunas,T., Tsvetkova,K., Matlashov,M., Truncaite,L., Morgan,R.D., Ivanov,N. V., et al. (2019) BREX system of Escherichia coli distinguishes self from non-self by methylation of a specific DNA site. Nucleic Acids Res., 47, 253-265.

https://doi.org/10.1093/nar/gky1125

22. Tai,C., Li,J., Ou,H., Li,X., Bi,D., Liu,M., Sun,J., Xie,Y. and Deng,Z. (2019) ICEberg 2.0: An updated database of bacterial integrative and conjugative elements. Nucleic Acids Res., 47.

https://doi.org/10.1093/nar/gky1123

23. Makarova,K.S., Anantharaman,V., Grishin,N. V., Koonin,E. V. and Aravind,L. (2014) CARF and WYL domains: Ligand-binding regulators of prokaryotic defense systems. Front. Genet., 5, 1-9.

https://doi.org/10.3389/fgene.2014.00102

24. Yen,M., Cairns,L.S. and Camilli,A. (2017) A cocktail of three virulent bacteriophages prevents Vibrio cholerae infection in animal models. Nat. Commun., 8, 14187.

https://doi.org/10.1038/ncomms14187

25. Melo,L.D.R., Oliveira,H., Pires,D.P., Dabrowska,K. and Azeredo,J. (2020) Phage therapy efficacy: a review of the last 10 years of preclinical studies. Crit. Rev. Microbiol., 46, 78-99.

https://doi.org/10.1080/1040841X.2020.1729695

http://www.ncbi.nlm.nih.gov/pubmed/32091280

26. Melo,L.D.R., Veiga,P., Cerca,N., Kropinski,A.M., Almeida,C., Azeredo,J. and Sillankorva,S. (2016) 
Development of a phage cocktail to control Proteus mirabilis catheter-associated urinary tract infections. Front. Microbiol., 7, 1-12.

https://doi.org/10.3389/fmicb.2016.01024

27. Mutreja,A., Kim,D.W., Thomson,N.R., Connor,T.R., Lee,J.H., Kariuki,S., Croucher,N.J., Choi,S.Y., Harris,S.R., Lebens,M., et al. (2011) Evidence for several waves of global transmission in the seventh cholera pandemic. Nature, 477.

https://doi.org/10.1038/nature10392

28. Spagnoletti,M., Ceccarelli,D., Rieux,A., Fondi,M., Taviani,E., Fani,R., Colombo,M.M., Colwell,R.R. and Balloux,F. (2014) Acquisition and Evolution of SXT-R391 Integrative Conjugative Elements in the SeventhPandemic Vibrio cholerae Lineage. 10.1128/mBio.01356-14.

https://doi.org/10.1128/mBio.01356-14

29. Waldor,M.K. and Mekalanos,J.J. (1996) Lysogenic conversion by a filamentous phage encoding cholera toxin. Science, 272, 1910-4.

http://www.ncbi.nlm.nih.gov/pubmed/8658163

30. O’Hara,B.J., Barth,Z.K., McKitterick,A.C. and Seed,K.D. (2017) A highly specific phage defense system is a conserved feature of the Vibrio cholerae mobilome. PLoS Genet., 13.

https://doi.org/10.1371/journal.pgen.1006838

http://www.ncbi.nlm.nih.gov/pubmed/28594826

31. Fomenkov,A., Lunnen,K.D., Zhu,Z., Anton,B.P., Wilson,G.G., Vincze,T. and Roberts,R.J. (2015) Complete Genome Sequence and Methylome Analysis of Bacillus Strain X1. 10.1128/genomeA.01593-14.

https://doi.org/10.1128/genomeA.01593-14

32. Garriss,G., Waldor,M.K. and Burrus,V. (2009) Mobile Antibiotic Resistance Encoding Elements Promote

Their Own Diversity. PLoS Genet, 5, 1000775.

https://doi.org/10.1371/journal.pgen.1000775

33. Rodríguez-Blanco Manuel Lemos Carlos R Osorio,A.L. Unveiling the pan-genome of the SXT/R391 family of ICEs: molecular characterisation of new variable regions of SXT/R391-like ICEs detected in

Pseudoalteromonas sp. and Vibrio scophthalmi. 10.1007/s10482-016-0716-3.

https://doi.org/10.1007/s10482-016-0716-3

34. Isaev,A., Drobiazko,A., Sierro,N., Gordeeva,J., Yosef,I., Qimron,U., Ivanov,N. V and Severinov,K. (2020) Phage T7 DNA mimic protein Ocr is a potent inhibitor of BREX defence. Nucleic Acids Res., 48, 5397-5406. https://doi.org/10.1093/nar/gkaa290

35. Samson,J.E., Magadán,A.H., Sabri,M. and Moineau,S. (2013) Revenge of the phages: Defeating bacterial defences. Nat. Rev. Microbiol., 11, 675-687.

https://doi.org/10.1038/nrmicro3096

http://www.ncbi.nlm.nih.gov/pubmed/23979432

36. Carraro,N., Sauvé,M., Matteau,D., Lauzon,G., Rodrigue,S. and Burrus,V. (2014) Development of pVCR94 $\mathrm{X}$ from Vibrio cholerae, a prototype for studying multidrug resistant IncA/C conjugative plasmids. Front. Microbiol., 5, 1-13.

https://doi.org/10.3389/fmicb.2014.00044

37. Burrus,V. and Waldor,M.K. (2003) Control of SXT Integration and Excision. J. Bacteriol., 185, 5045-5054. https://doi.org/10.1128/JB.185.17.5045-5054.2003

38. Beaber,J.W., Hochhut,B. and Waldor,M.K. (2004) SOS response promotes horizontal dissemination of antibiotic resistance genes. Nature, 427, 72-74.

https://doi.org/10.1038/nature02241

39. Seed,K.D., Bodi,K.L., Kropinski,A.M., Ackermann,H.W., Calderwood,S.B., Qadri,F. and Camilli,A. (2011) Evidence of a dominant lineage of Vibrio cholerae-specific lytic bacteriophages shed by cholera patients over a 10-year period in Dhaka, Bangladesh. MBio, 10.1128/mBio.00334-10.

https://doi.org/10.1128/mBio.00334-10

http://www.ncbi.nlm.nih.gov/pubmed/21304168

40. Baddam,R., Sarker,N., Ahmed,D., Mazumder,R., Abdullah,A., Morshed,R., Hussain,A., Begum,S., 
Shahrin,L., Khan,A.I., et al. (2020) Genome Dynamics of Vibrio cholerae Isolates Linked to Seasonal Outbreaks of Cholera in Dhaka, Bangladesh. MBio, 11, 1-14.

https://doi.org/10.1128/mBio.03339-19

41. Aminov,R., Kumar,P., Kushwaha Bhardwaj,A., Ramamurthy,T., Sarkar,A., Morita,D., Ghosh,A., Chowdhury,G., Mukhopadhyay,A.K. and Okamoto,K. (2019) Altered Integrative and Conjugative Elements (ICEs) in Recent Vibrio cholerae O1 Isolated From Cholera Cases, Kolkata, India. 10.3389/fmicb.2019.02072. https://doi.org/10.3389/fmicb.2019.02072

42. Balado,M., Lemos,M.L. and Osorio,C.R. (2013) Integrating conjugative elements of the SXT/R391 family from fish-isolated Vibrios encode restriction-modification systems that confer resistance to bacteriophages. FEMS Microbiol. Ecol., 83, 457-467.

https://doi.org/10.1111/1574-6941.12007

43. Gaba,S. and Ebert,D. (2009) Time-shift experiments as a tool to study antagonistic coevolution. Trends Ecol. Evol., 24, 226-232.

https://doi.org/10.1016/j.tree.2008.11.005

http://www.ncbi.nlm.nih.gov/pubmed/19201504

44. Gómez,P. and Buckling,A. (2011) Bacteria-phage antagonistic coevolution in soil. Science (80-. )., 332, 106-109.

https://doi.org/10.1126/science. 1198767

http://www.ncbi.nlm.nih.gov/pubmed/21454789

45. Papkou,A., Guzella,T., Yang,W., Koepper,S., Pees,B. and Schalkowski,R. (2019) The genomic basis of Red Queen dynamics during rapid reciprocal host - pathogen coevolution. 116, 923-928.

https://doi.org/10.1073/pnas.1810402116

46. Betts,A., Kaltz,O. and Hochberg,M.E. (2014) Erratum: Contrasted coevolutionary dynamics between a bacterial pathogen and its bacteriophages(Proceedings of the National Academy of Sciences of the United States of America (2014) 111, 30 (11109-11114) DOI: 10.1073/pnas.1406763111). Proc. Natl. Acad. Sci. U. S. A., 111, 15279.

https://doi.org/10.1073/pnas.1418380111

47. Woolhouse,M.E.J., Webster,J.P., Domingo,E., Charlesworth,B. and Levin,B.R. (2002) Biological and biomedical implications of the co-evolution of pathogens and their hosts. Nat. Genet., 32, 569-577.

https://doi.org/10.1038/ng1202-569

http://www.ncbi.nlm.nih.gov/pubmed/12457190

48. Hall,J.P.J., Harrison,E., Pärnänen,K., Virta,M. and Brockhurst,M.A. (2020) The Impact of Mercury Selection and Conjugative Genetic Elements on Community Structure and Resistance Gene Transfer. Front. Microbiol., 11, 1-14.

https://doi.org/10.3389/fmicb.2020.01846

49. Oliveira,P.H., Touchon,M. and Rocha,E.P.C. (2016) Regulation of genetic flux between bacteria by restriction-modification systems. Proc. Natl. Acad. Sci. U. S. A., 113, 5658-5663.

https://doi.org/10.1073/pnas.1603257113

http://www.ncbi.nlm.nih.gov/pubmed/27140615

50. Dalia,A.B., Seed,K.D., Calderwood,S.B. and Camilli,A. A globally distributed mobile genetic element inhibits natural transformation of Vibrio cholerae. 10.1073/pnas.1509097112.

https://doi.org/10.1073/pnas.1509097112

51. Alexandrova,L., Haque,F., Rodriguez,P., Marrazzo,A.C., Grembi,J.A., Ramachandran,V., Hryckowian,A.J., Adams,C.M., Siddique,M.S.A., Khan,A.I., et al. (2019) Identification of Widespread Antibiotic Exposure in Patients with Cholera Correlates with Clinically Relevant Microbiota Changes. J. Infect. Dis., 220, $1655-1666$. https://doi.org/10.1093/infdis/jiz299

http://www.ncbi.nlm.nih.gov/pubmed/31192364

52. Takano,S., Fukuda,K., Koto,A. and Id,R.M. (2019) A novel system of bacterial cell division arrest implicated in horizontal transmission of an integrative and conjugative element. 10.1371/journal.pgen. 1008445. https://doi.org/10.1371/journal.pgen.1008445 
53. Alam,M., Sultana,M., Nair,G.B., Sack,R.B., Sack,D.A., Siddique,A.K., Ali,A., Huq,A. and Colwell,R.R. (2006) Toxigenic Vibrio cholerae in the aquatic environment of Mathbaria, Bangladesh. Appl. Environ.

Microbiol., 72, 2849-2855.

https://doi.org/10.1128/AEM.72.4.2849-2855.2006

http://www.ncbi.nlm.nih.gov/pubmed/16597991

54. Mckitterick,A.C. and Seed,K.D. Anti-phage islands force their target phage to directly mediate island excision and spread. 10.1038/s41467-018-04786-5.

https://doi.org/10.1038/s41467-018-04786-5

55. Mckitterick,A.C., Legault,K.N., Angermeyer,A., Alam,M. and Seed,K.D. Competition between mobile genetic elements drives optimization of a phage-encoded CRISPR-Cas system: insights from a natural arms race. $10.1098 /$ rstb.2018.0089.

https://doi.org/10.1098/rstb.2018.0089

56. Bankevich,A., Nurk,S., Antipov,D., Gurevich,A.A., Dvorkin,M., Kulikov,A.S., Lesin,V.M., Nikolenko,S.I., Pham,S., Prjibelski,A.D., et al. (2012) SPAdes: A new genome assembly algorithm and its applications to single-cell sequencing. J. Comput. Biol., 19, 455-477.

https://doi.org/10.1089/cmb.2012.0021

http://www.ncbi.nlm.nih.gov/pubmed/22506599

57. El-Gebali,S., Mistry,J., Bateman,A., Eddy,S.R., Luciani,A., Potter,S.C., Qureshi,M., Richardson,L.J., Salazar,G.A., Smart,A., et al. (2019) The Pfam protein families database in 2019. Nucleic Acids Res., 47, D427-D432.

https://doi.org/10.1093/nar/gky995

http://www.ncbi.nlm.nih.gov/pubmed/30357350

58. Roberts,R.J., Vincze,T., Posfai,J. and Macelis,D. (2015) REBASE-a database for DNA restriction and modification: Enzymes, genes and genomes. Nucleic Acids Res., 43, D298-D299.

https://doi.org/10.1093/nar/gku1046

http://www.ncbi.nlm.nih.gov/pubmed/25378308

59. Roy,A., Kucukural,A. and Zhang,Y. (2010) I-TASSER: A unified platform for automated protein structure and function prediction. Nat. Protoc., 5, 725-738.

https://doi.org/10.1038/nprot.2010.5

60. Seed, K. D., Lazinski, D. W., Calderwood, S. B., \& Camilli, A. (2013). A bacteriophage encodes its own CRISPR/Cas adaptive response to evade host innate immunity. Nature, 494.

https://doi.org/10.1038/nature11927

61. Barth, Z. K., Netter Z, Angermeyer A, Bhardwaj P and Seed KD. 2020. A family of viral satellites manipulates invading virus gene expression and affects cholera toxin mobilization. mSystems. 5:e00358-20.

\section{Acknowledgments:}

The authors are especially thankful to icddr,b hospital and lab staff for support. The authors thank members of the Seed lab for critical feedback and thoughtful discussion regarding this manuscript and Vivek Mutalik for sharing the E. coli phages. Funding: This work was supported by the National Institute of Allergy and Infectious Diseases (grants R01AI127652 and R01AI153303 to K.D.S.). K.D.S. is a Chan Zuckerberg Biohub Investigator and holds an Investigators in the Pathogenesis of Infectious Disease Award from the Burroughs Wellcome Fund. K.N.L. was supported by an NSF Graduate Research Fellowship (Fellow ID no. 2017242013). icddr,b gratefully acknowledges the following donors which provide unrestricted support: Government of the People's Republic of Bangladesh, Global Affairs Canada (GAC), Swedish International Development Cooperation Agency (SIDA), and the Department for International Development, UK Aid. Author contributions: K.N.L: Conceptualization, Investigation, Writing - Original draft preparation. S.G.H.: Investigation, Visualization. A.A.: Formal analysis, Software. A.C.M.: Investigation. F.T.J.: Resources, Visualization. M.S.: Resources, Project Administration. T. A: Resources, Supervision. M.A.: Resources, 
Supervision. K.D.S.: Conceptualization, Investigation, Writing - Review \& Editing, Project administration, Funding acquisition. All authors discussed the results, commented on and approved the final manuscript.

Competing interests: K.D.S. is a scientific advisor for Nextbiotics, Inc. Data and materials availability: All data are available in the manuscript or the supplementary materials.

Supplementary Materials:

Materials and Methods

Figures S1-S9

Tables S1-S6 
A

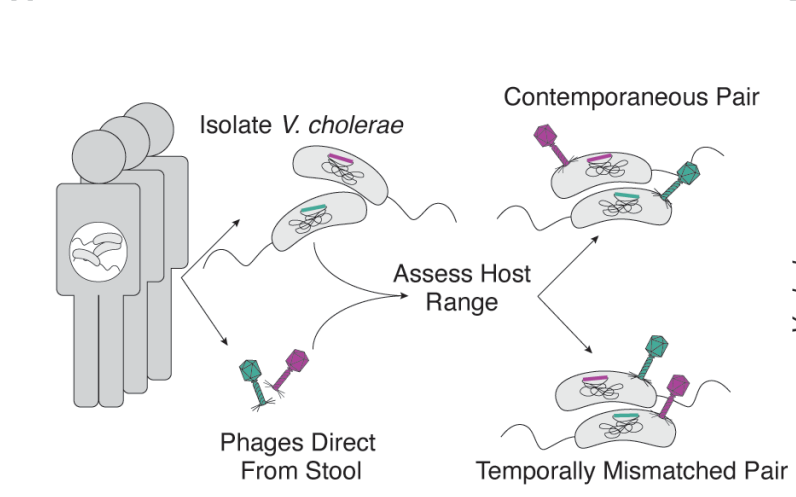

D

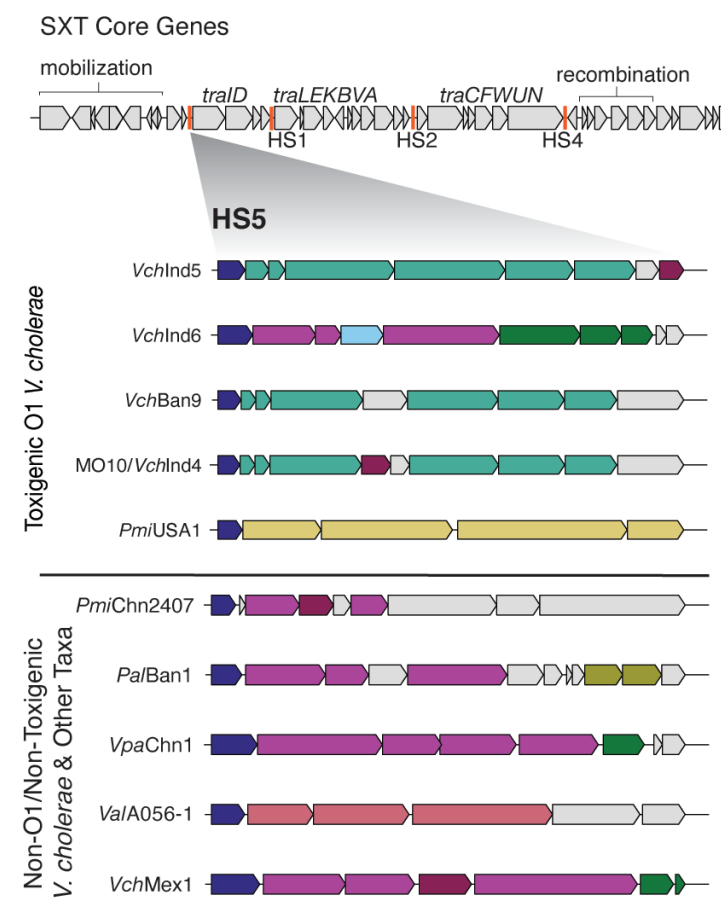

B

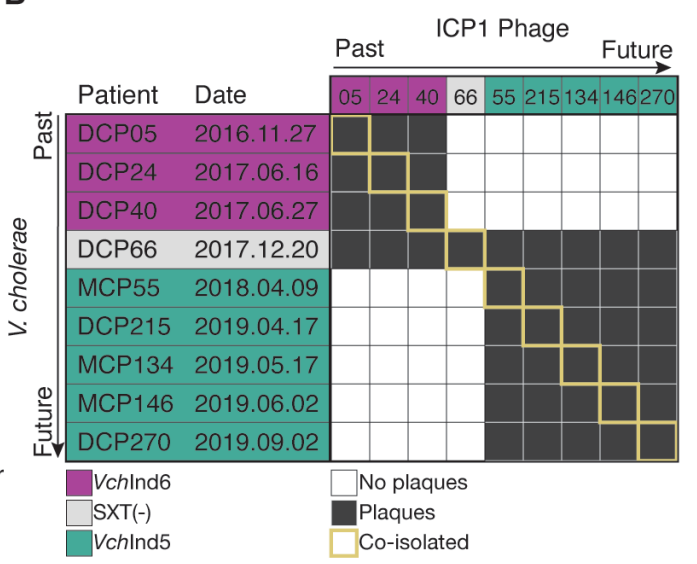

C

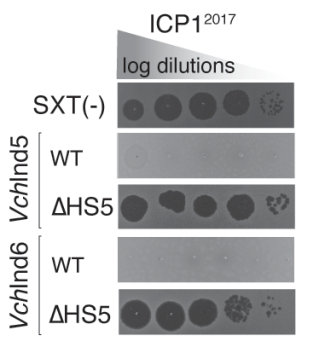

E

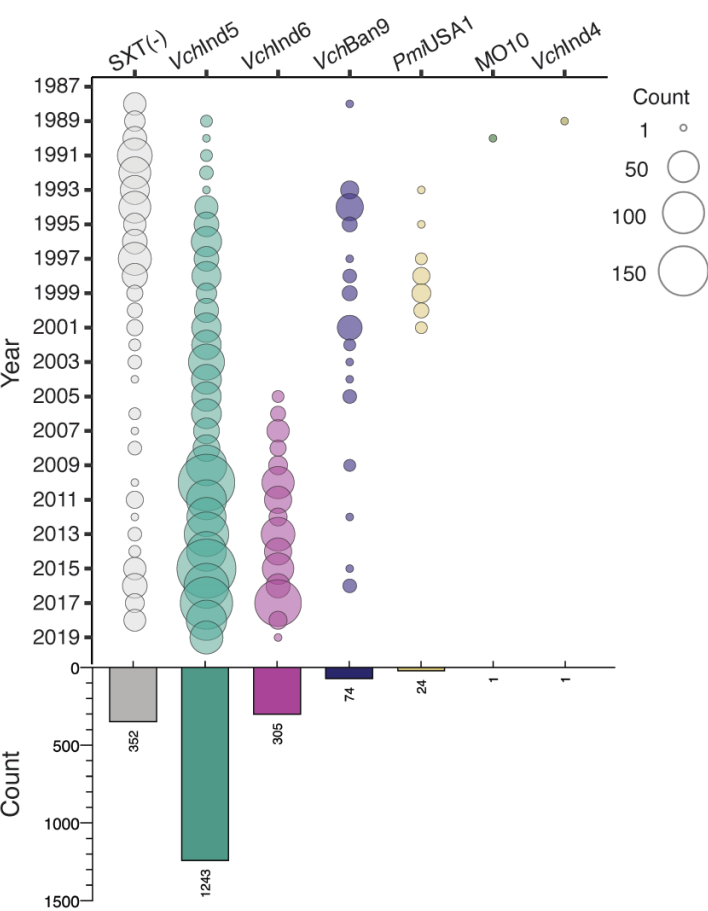

Fig. 1. The prevalence and identity of SXT ICEs in clinical $V$. cholerae determines susceptibility to phages. (A) Schematic of time-shift experiments. ICP1 phage in cholera patient stool samples were probed for ability to productively infect isolates of $V$. cholerae from contemporaneous samples or from the past or future. Matching colors denote contemporaneously isolated $V$. cholerae and phage pairs. (B) V. cholerae (vertical axis) susceptibility matrix to ICP1 phages from past or future patients (top horizontal axis), where colors denote the presence of the SXT ICE (as identified through whole genome sequencing of patient isolates) in $V$. cholerae from the patient stool sample. ICE $V c h$ Ind6 $V$. cholerae were susceptible to phages shed from patients in which ICE $V c h$ Ind6 $V$. cholerae was recovered, but restricted phages from patients in which SXT(-) or ICE $V c h$ Ind5 $V$. cholerae was recovered. The SXT(-) isolate was only susceptible to co-isolated phage. ICEVchInd5 V. cholerae isolates restricted phages from the past but were susceptible to phages from other patients in which ICEVchInd5 $V$. cholerae was recovered. (C) Tenfold dilutions of ICP $1^{2017}$ (isolated from patient DCP24, propagated on SXT(-) V. cholerae) spotted on an SXT(-) V. cholerae host or exconjugant with the SXT ICE indicated (bacterial lawns in gray, zones of killing are shown in black). (D) Genomic organization of SXT ICE core genes (modified from (19)), with core function labeled above, tra genes encoding functions related to conjugation and 
hotspot regions (HS) denoted by orange lines (not to scale). Diverse anti-phage systems are encoded in hotspot 5 of SXT ICEs found in toxigenic O1 V. cholerae (top) and other hosts (bottom). (E) Temporal distribution of SXT ICEs in toxigenic O1 V. cholerae between 1987-2019 $(n=2,000)$. The size of the circle scales with the number of genomes analyzed per year, with total counts for each SXT ICE denoted below. 
bioRxiv preprint doi: https://doi.org/10.1101/2020.12.16.423150; this version posted December 21, 2020. The copyright holder for this preprint (which was not certified by peer review) is the author/funder, who has granted bioRxiv a license to display the preprint in perpetuity. It is made available under aCC-BY-ND 4.0 International license.

A

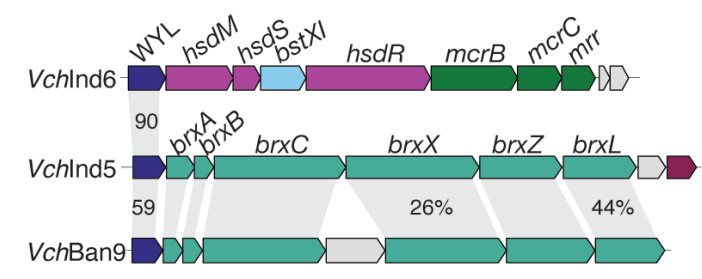

B

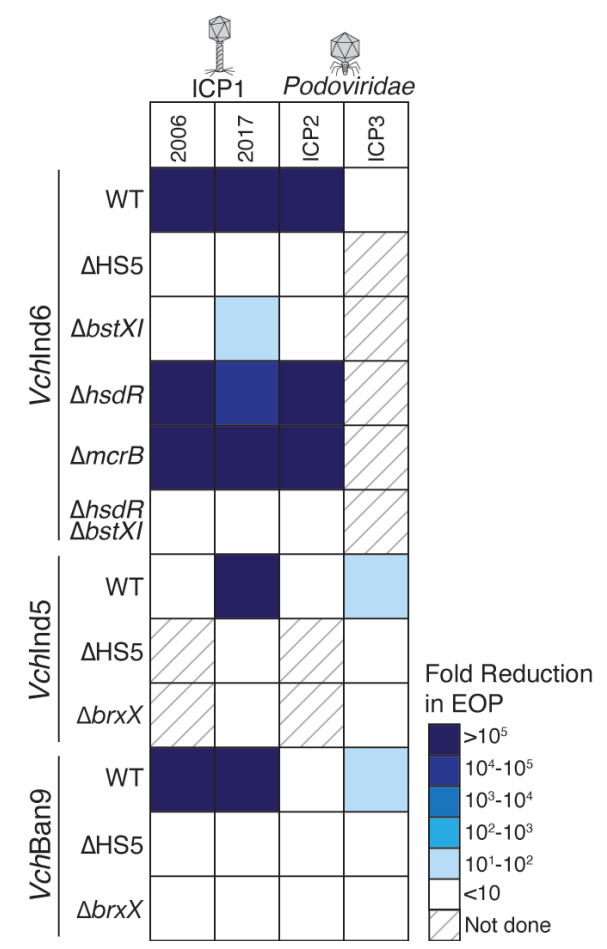

C

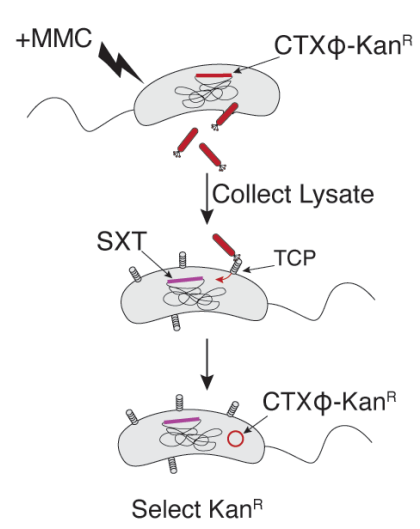

$E$

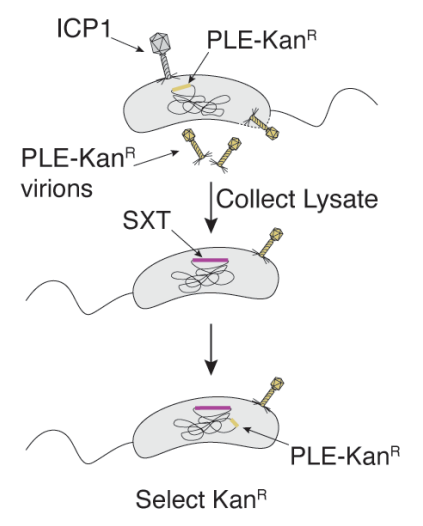

D

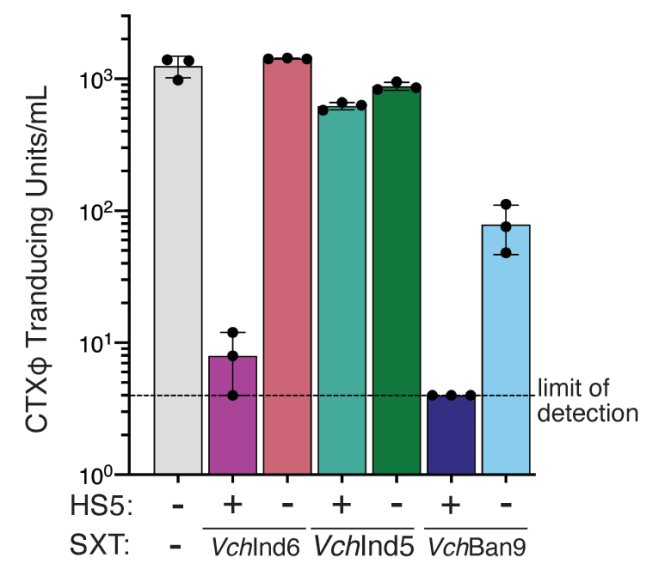

$\mathbf{F}$

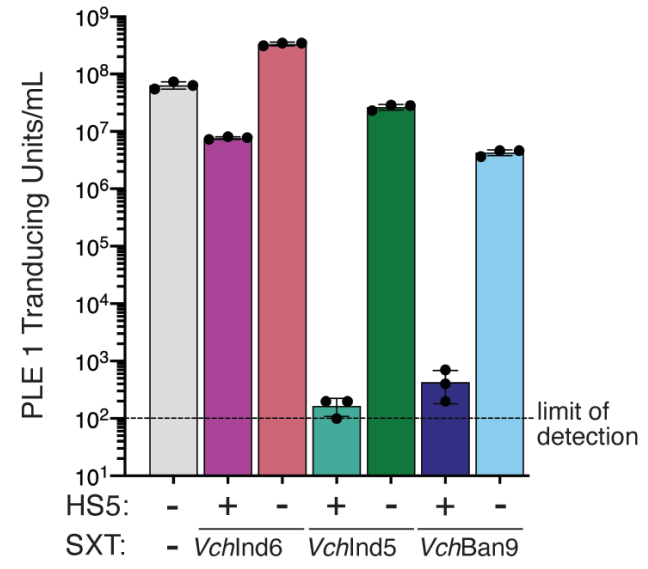

Fig. 2. SXT ICEs confer resistance to phages and mobile elements in $\boldsymbol{V}$. cholerae. (A) Genomic organization of hotspot 5 of the three dominant SXT ICEs in toxigenic O1 $V$. cholerae labeled according to homologous genes identified by REBASE/PFAM searches. Color scheme is identical to Fig. 1D. Shading denotes proteins with same predicted function; Percent identity is indicated for genes encoding proteins that share $>20 \%$ amino acid identity, if unlabeled percent identity is $<20 \%$. (B) Fold reduction in the efficiency of plaquing (EOP) of lytic vibriophages afforded by SXT ICEs under native expression levels. When the SXT ICE did not reduce EOP, deletions were not tested. Source data is shown in Fig. S2. (C) Schematic representation of the filamentous prophage CTX $\phi$ transduction assay. (D) Transduction of CTX $\phi$ into $V$. cholerae possessing each SXT ICE and derivatives with hotspot 5 deletions. (E) Schematic representation of PLE transduction. (F) Transduction of PLE 1 into $V$. cholerae possessing each SXT ICE and derivatives lacking hotpot 5. 
bioRxiv preprint doi: https://doi.org/10.1101/2020.12.16.423150; this version posted December 21,2020 . The copyright holder for this preprint (which was not certified by peer review) is the author/funder, who has granted bioRxiv a license to display the preprint in perpetuity. It is made available under aCC-BY-ND 4.0 International license.

A

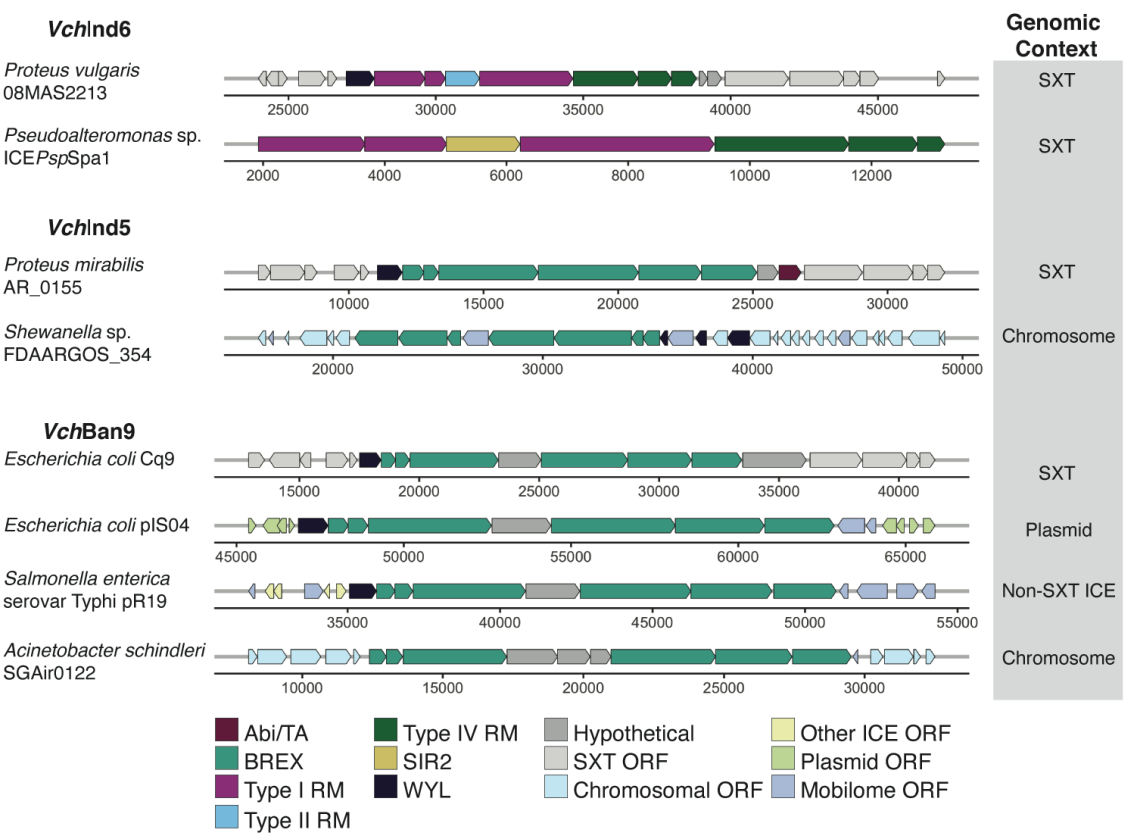

B

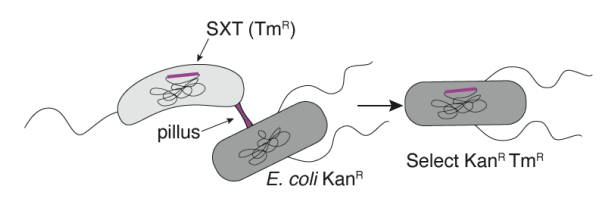

C

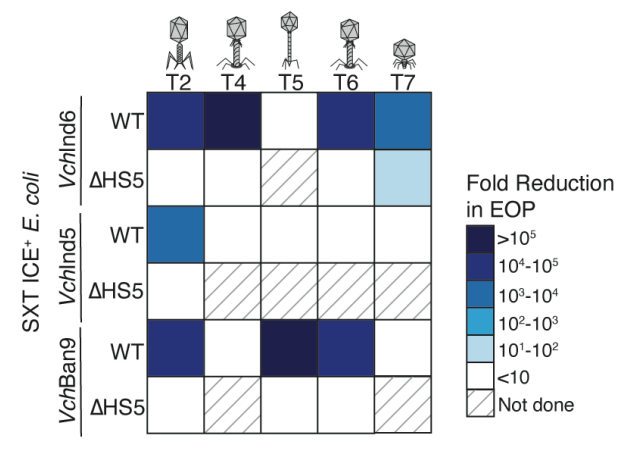

Fig. 3. Hotspot 5 from $V$. cholerae SXT ICEs occur in other Gammaproteobacteria and confer phage resistance upon conjugation into E.coli. (A) Gene maps depicting instances of hotspot 5 genes (from the start of the WYL-domain protein to the end of the last gene) from ICEVchInd6, ICEVchInd5 and ICEVchBan9 found outside of $V$. cholerae, identified using BLASTn (shown are hotspot 5 genes with $>90 \%$ nucleotide identity across $>75 \%$ of the query). The genomic context of the hotspot 5 genes identified is indicated in the shaded box to the right. (B) Schematic depicting SXT ICE conjugative transfer from $V$. cholerae into Escherichia coli. (C) Fold-reduction in the efficiency of plaquing (EOP) of lytic coliphages mediated by SXT ICEs and corresponding hotspot 5 deletions in E. coli under native expression levels. When the SXT ICE did not reduce EOP, the deletion was not tested. Source data is shown in Fig. S5. 
A

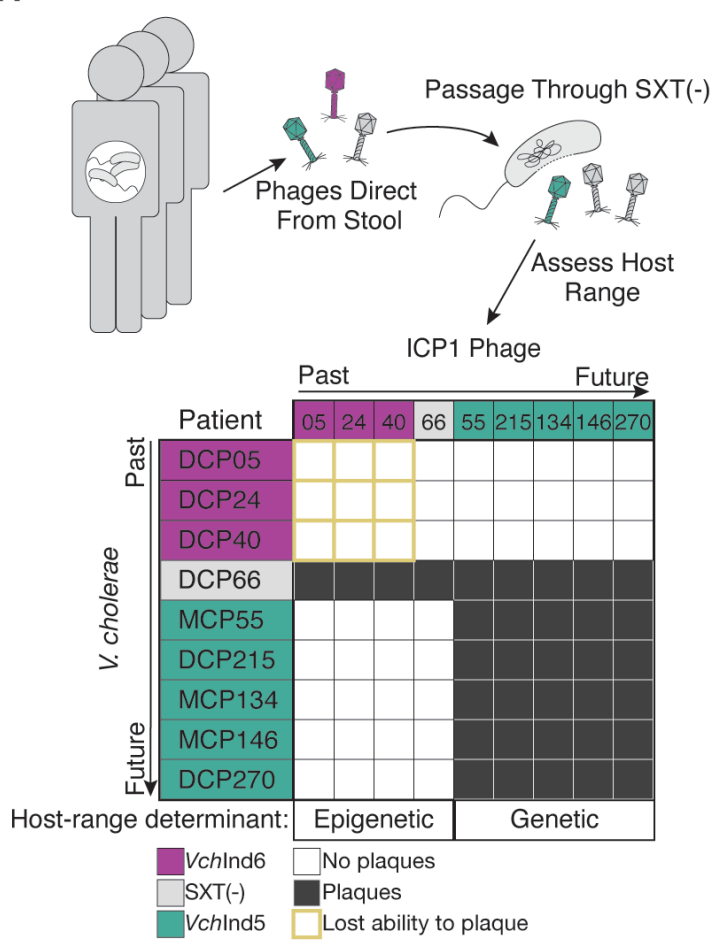

B

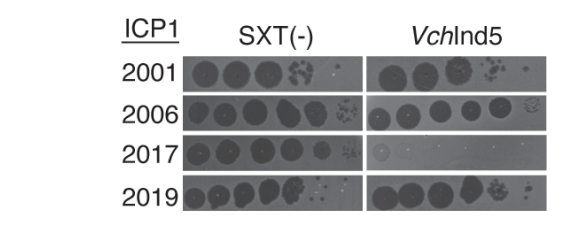

\section{C}
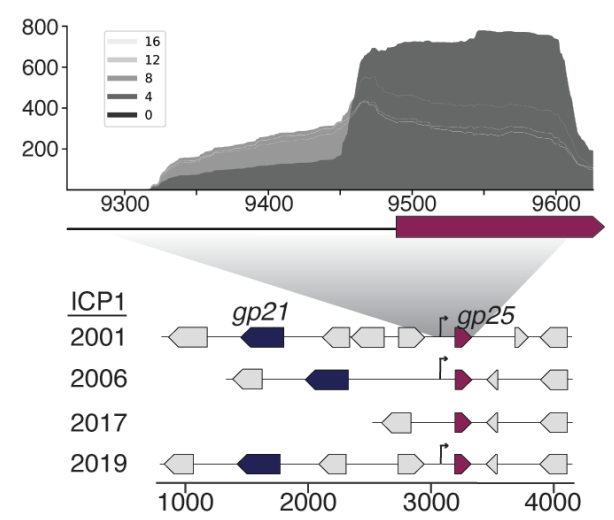

D

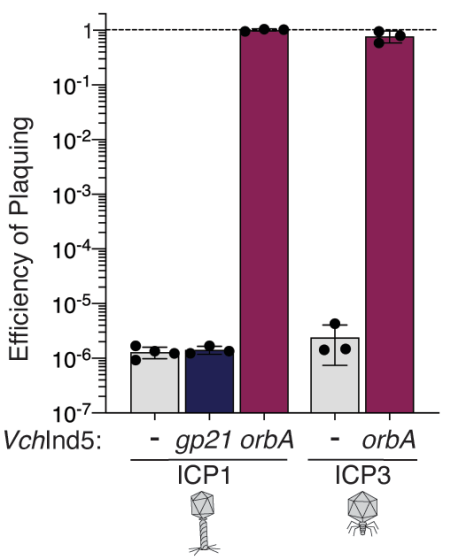

E

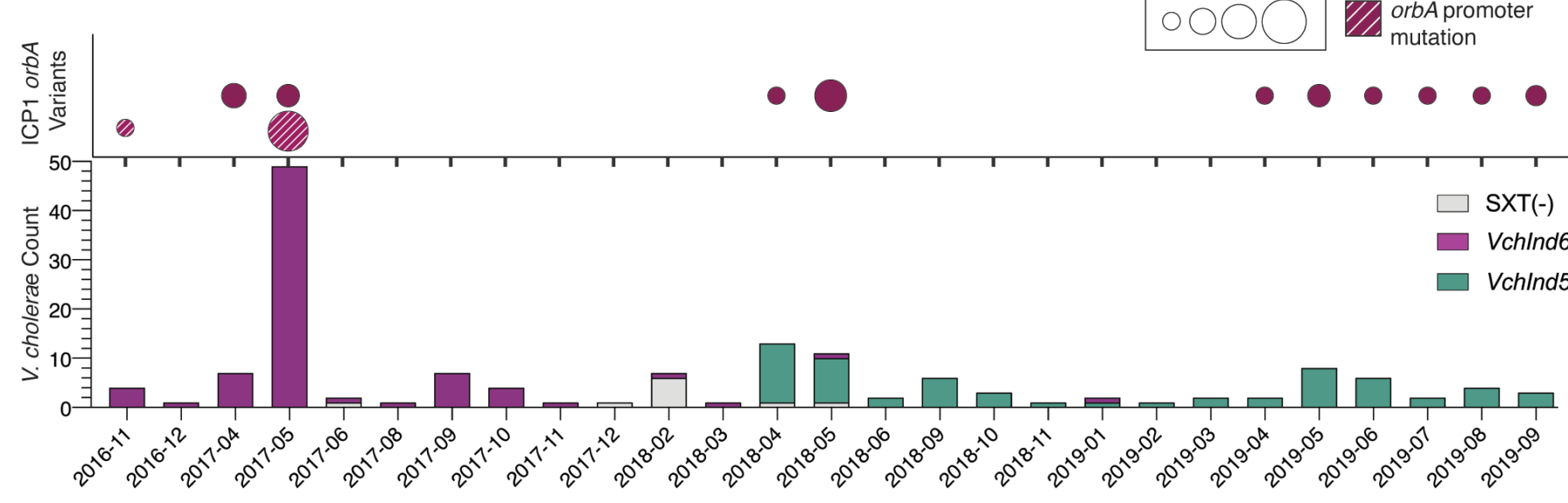

Fig. 4. Clinical ICP1 overcomes co-circulating SXT ICEs through acquisition of epigenetic modification as well as an anti-BREX protein OrbA. (A) ICP1 isolates from cholera-patient stool samples were propagated on an SXT(-) strain and then probed for ability to infect clinical $V$. cholerae isolates. ICP1 isolates from ICE $V$ chInd6(+) stool samples lost the ability to infect co-circulating $V$. cholerae upon passage through an SXT(-) host indicative of initial escape through epigenetic modification. ICP1 isolates from ICE $V c h$ Ind5(+) patients retained infectivity of co-circulating $V$. cholerae after passage through an SXT(-) host, suggestive of a genetic means of overcoming ICE $V c h$ Ind5. (B) Tenfold dilutions of ICP1 isolates spotted on SXT(-) $V$. cholerae or an isogenic exconjugant of ICE $V$ chInd5 identified ICP ${ }^{2017}$ (from patient DCP24) as uniquely inhibited by ICEVchInd5 (bacterial lawns in gray, zones of killing are shown in black). (C) Comparative genomics of ICP1 isolates showing that ICP $1^{2017}$ lacks gp21-24 and the promoter for gp 25, as identified through RNA-seq of ICP1 ${ }^{2006}$ (top, displaying average read coverage over the course of infection, reads are color coded by time point in minutes post infection). (D) Ectopic expression of $g p 21$ and $g p 25$ in ICEVchInd5(+) $V$. cholerae: gp25 (designated orbA for "overcome restriction by BREX") but not gp21 restores plaquing by ICP $1^{2017}$ as well the unrelated lytic phage ICP3. (E) Sampling and whole genome sequencing of $151 \mathrm{~V}$. cholerae isolates and 44 ICP1 isolates from cholera patient stool samples in Bangladesh, depicting isolate counts (y-axis) over time, showing the transition from ICE $V c h$ Ind6 to SXT(-) to ICE $V c h \operatorname{Ind5(+)} V$. 
bioRxiv preprint doi: https://doi.org/10.1101/2020.12.16.423150; this version posted December 21, 2020. The copyright holder for this preprint (which was not certified by peer review) is the author/funder, who has granted bioRxiv a license to display the preprint in perpetuity. It is made available under aCC-BY-ND 4.0 International license.

cholerae. ICP1 isolates with the gp25 promoter mutation were only isolated from patients in which we recovered ICEVchInd6(+) V. cholerae, and have not been isolated since the re-emergence of ICE $V c h \operatorname{Ind} 5(+) V$. cholerae. 
bioRxiv preprint doi: https://doi.org/10.1101/2020.12.16.423150; this version posted December 21, 2020. The copyright holder for this preprint (which was not certified by peer review) is the author/funder, who has granted bioRxiv a license to display the preprint in perpetuity. It is made available under aCC-BY-ND 4.0 International license.

A

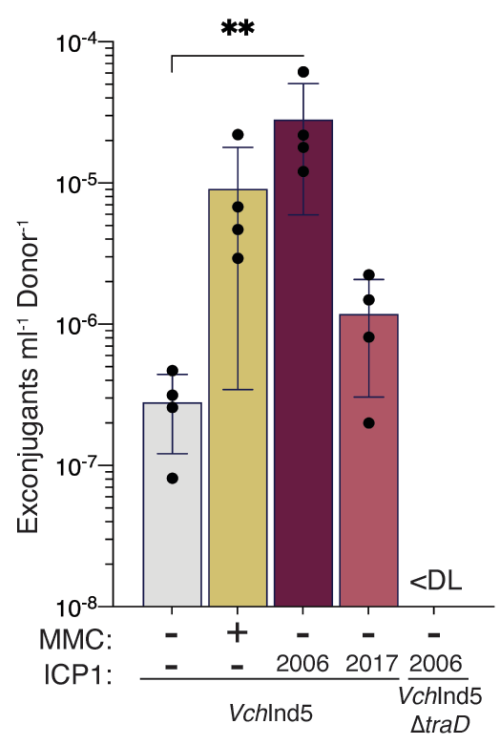

B

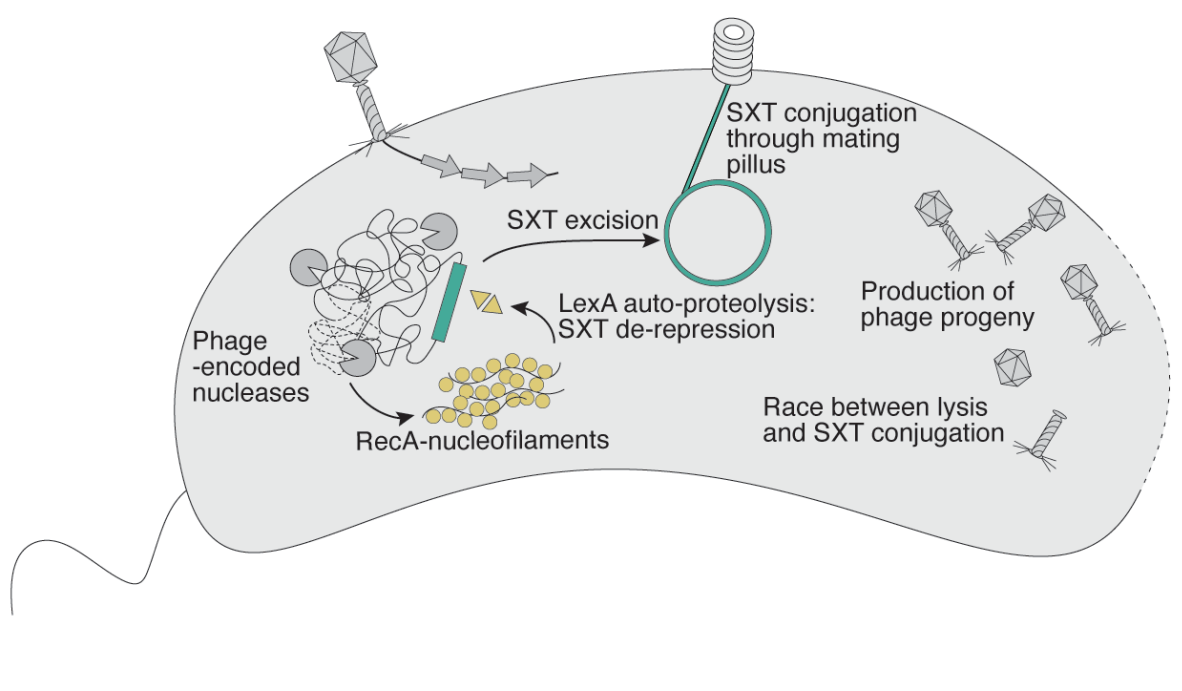

Fig. 5. ICP1 infection increases SXT ICE conjugation frequency. (A) Conjugation frequency from a donor $V$. cholerae mock treated (LB), mitomycin C (MMC) treated or infected by ICP ${ }^{2006}$ (unrestricted phage) or ICP $1^{2017}$ (restricted phage). Asterisks denotes significance by ANOVA p-value $<0.01$. No exconjugants were detected from a phage infected donor lacking traD (DL is the limit of detection). (B) Model representing a possible mechanism of ICP1-stimulated SXT conjugation: Upon infection, ICP1-encoded nucleases degrade the $V$. cholerae genome, triggering SXT de-repression through the SOS response, resulting in the SXT ICE escaping the lysing host cell through conjugation. 